\title{
The Role of Fractional Excretion of Uric Acid in the Differential Diagnosis of Hypotonic Hyponatraemia in Patients with Diuretic Therapy
}

\author{
Vincenzo Bassi ${ }^{1}$, Olimpia Fattoruso ${ }^{2}$ \\ 1. Internal Medicine, San Giovanni Bosco Hospital, ASL Napoli 1 Centro, Naples, ITA 2. Pathology, San Giovanni Bosco \\ Hospital, ASL Napoli 1 Centro, Naples, ITA
}

Corresponding author: Vincenzo Bassi, dr_bassi@inwind.it

\begin{abstract}
Hyponatraemia is the most common electrolyte imbalance found in hospital population and worldwide thiazide and loop-diuretics are among the most widely used drugs. Syndrome of inappropriate antidiuresis diagnosis (SIAD) is complicated in the presence of diuretic therapy due to the misleading clinical assessment of the extracellular volume status, and in order to make SIAD diagnosis it is often necessary to withdraw diuretic therapy. Our study aimed to investigate the diagnostic role of these alternative markers of volume status, serum uric acid (sUA) and fractional excretion of uric acid (FEUA), in hyponatraemic patients treated with different diuretic drugs. Eighty-nine patients were enrolled with the diagnosis of SIAD, diuretic-induced hyponatremia (DIH, treated with furosemide and potassium canrenoate) or thiazide-induced hyponatremia ( $\mathrm{TIH}$, treated with hydrochlorothiazide, metolazone or indapamide) and investigated with receiver operating characteristic analysis and a sensitivity test. Our results show that FEUA discriminated better than sUA between SIAD and DIH patients (area under curve $0.96,<0.001$ vs. 0.88 , $<0.001)$ while it was a poor marker to discriminate between SIAD and TIH (0.65, NS vs. 0.67, NS). In conclusions, FEUA is an excellent marker to discriminate SIAD vs. sodium depleted patients treated with furosemide and/or potassium canrenoate while the diuretic withdrawal, beyond obtaining a serum $\mathrm{Na}$ normalization, is still mandatory for differential diagnosis of sodium depleted patients affected by thiazideinduced hyponatraemia.
\end{abstract}

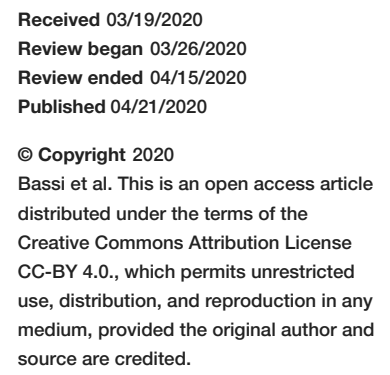

Categories: Endocrinology/Diabetes/Metabolism, Internal Medicine

Keywords: syndrome of inappropriate antidiuresis, hyponatraemia, diuretics, thiazide diuretics, furosemide, potassium canrenoate, syndrome of inappropriate antidiuretic hormone secretion

\section{Introduction}

Hyponatraemia is a frequent electrolyte imbalance occurring in up to $25-30 \%$ of hospitalized patients where syndrome of inappropriate antidiuresis (SIAD) is the most common etiology present in nearly $35 \%$ of hyponatremic inpatients $[1,2]$. The actual guidelines suggest different algorithms to help the physicians in the differential diagnosis of hypotonic hyponatraemia, but a potential bias is coming from the clinical assessment of the volemic status, especially in diuretic-treated patients [3-5].

Worldwide thiazide and loop-diuretics are among the most widely used drugs blocking the respective ioncotransporters at different sites with inhibition of tubular Na reabsorption and increase of urine Na and water excretion. The SIAD diagnosis is complicated in the presence of diuretic therapy due to the misleading clinical assessment of the extracellular volume status [6]. In many clinical trials, the presence of diuretics arbitrarily excluded the diagnosis of SIAD in hyponatremic patients [7, 8]. In order to make SIAD diagnosis it is often necessary to withdraw diuretic therapy up to 10 days [9].

Fenske et al. demonstrated that the fractional excretion of uric acid (FEUA) could discriminate between SIAD and diuretic-induced hyponatraemia in these confounding patients but only $13 \%$ of enrolled patients were treated with thiazide therapy [5].

The aim of our study was to investigate the diagnostic role of these alternative markers of volume status, serum uric acid (sUA) and FEUA, in hyponatremic patients treated with different diuretic drugs.

\section{Materials And Methods}

Retrospectively, between March 2016 and October 2018, 122 patients, older than 18 years, presenting a serum sodium concentration $<130 \mathrm{mEq} / \mathrm{L}$, serum osmolality $<280 \mathrm{mEq} / \mathrm{Kg}$, were consecutively identified and 89 patients enrolled with the diagnosis of SIAD or diuretic-induced hyponatraemia. Serum hyponatraemia was confirmed using the hemogasanalysis study (direct measurement) to exclude bias of dilution.

Further eligibility criteria were a normal kidney, thyroid and adrenal function, urine osmolality >100 
$\mathrm{mEq} / \mathrm{kg}$, a sufficient dietary daily intake (at least $10 \mathrm{mEq} / \mathrm{kg}$ as solutes), no polydipsia story and, if treated, a diuretic therapy (hydrochlorothiazide, metolazone, indapamide, furosemide and potassium canrenoate) longer than three months. The presence of liver cirrhosis and acute neurological diseases (potentially complicated by cerebral salt wasting syndrome] were non-eligibility criteria in consideration of the interference with FEUA values [10, 11].

Particular attention was paid to the medical anamnesis and the pharmacotherapy of the enrolled patients. The effective arterial blood volume (EABV) status was investigated using the parameters of change of pulse rate and blood pressure on supine and upright body position in enrolled patients [12]. Hypervolemic patients, usually with a congestive heart failure diagnosis, were identified with a clinical examination of edema presence. The normalization of serum $\mathrm{Na}$ after the withdrawal of diuretic therapy determined the diagnosis of diuretic-induced hyponatraemia. In doubtful cases a fluid challenge test ( $2 \mathrm{~L}$ isotonic saline in $24 \mathrm{~h}$ ) was performed and a serum sodium increase $>5 \mathrm{mEq} / \mathrm{L}$ with a $\triangle \mathrm{FENa}<0.5 \%$ identified a mild hypovolemic status of a non-SIAD patient.

Laboratory parameters were tested with automated clinical analysis, using ion-selective electrodes (indirect measurement, COBAS 6000 Analyzer Series, Roche, Switzerland). A blood sample was taken in the morning contemporary to a urine spot sample to test $\mathrm{Na}$, K, uric acid, glucose, blood urea nitrogen (BUN), creatinine and then calculate the different fractional excretions (FENa, FEUA).

We identified a SIAD and a non-SIAD diuretic-induced hyponatraemia group.

SIAD diagnosis was based on the classical Schwartz and Bartter criteria such as a serum hyponatraemia $<130$ $\mathrm{mEq} / \mathrm{L}$, a serum osmolality $<280 \mathrm{mEq} / \mathrm{kg}$ (either measured or calculated with the formula: Posm $=\mathrm{Na}[\mathrm{mEq} / \mathrm{L}$ ] $\mathrm{x} 1.86+$ glucose $/ 18[\mathrm{mEq} / \mathrm{L}]+\mathrm{BUN} / 6[\mathrm{mEq} / \mathrm{L}]+9)$, clinical euvolemia, urine sodium concentration $>30$ $\mathrm{mEq} / \mathrm{L}$, urine osmolality $>100 \mathrm{mEq} / \mathrm{kg}$ (either measured or calculated with the formula: Posm $=\mathrm{Na}[\mathrm{mEq} / \mathrm{L}] \mathrm{x}$ $1.86+$ glucose $/ 18[\mathrm{mEq} / \mathrm{L}]+\mathrm{BUN} / 6[\mathrm{mEq} / \mathrm{L}]+9)[13]$.

In the presence of coexistent diuretic therapy the persistence of hyponatraemia $<130 \mathrm{mEq} / \mathrm{L}$ to the diuretic withdrawal confirmed the diagnosis of SIAD. Instead, the non-SIAD diuretic-induced hyponatraemia diagnosis was based on hypovolemic or hypervolemic clinical status and normalization of hyponatraemia after diuretic withdrawal.

The non-SIAD diuretic-induced group was divided into two subgroups:

Diuretic-induced hyponatraemia (DIH, patients treated with furosemide and/or potassium canrenoate) and thiazide-induced hyponatraemia group (TIH, patients treated with hydrochlorothiazide, metolazone or indapamide).

All data analysed retrospectively were collected as part of routine diagnosis. Written informed consent was obtained from all the patients before participation.

\section{Statistical analysis}

The means of different groups were tested with a nonparametric Kruskal-Wallis test. Group comparisons between patients with and without SIAD were performed with the Student's test after testing for equality of variances with Levene's test.

The accuracy of SIADH diagnosis vs. diuretic-induced hyponatraemia group was tested using receiver operating characteristic (ROC) analysis calculating area under the curve (AUC) by the nonparametric trapezoidal rule with $95 \%$ confidence interval (CI). A sensitivity analysis to identify the optimal cutoff points was performed using Wilson/Brown method. The statistical analysis was performed with Prism 8.3.0 program (GraphPad Software, San Diego, CA, USA).

\section{Results}

The characteristics of investigated patients, different etiologies and treatments are shown in Table 1 . 


\section{Cureus}

\begin{tabular}{|c|c|c|c|}
\hline & SIAD Group (n = 42) & DIH Group $(n=25)$ & TIH Group ( $n=22)$ \\
\hline Age & $69.4 \pm 11.5$ & $72.0 \pm 5.1$ & $77.3 \pm 7.2$ \\
\hline $\operatorname{Sex}(M / F)$ & 23/19 & $12 / 13$ & 10/12 \\
\hline \multicolumn{4}{|l|}{ Etiology SIAD } \\
\hline Neoplasia & 17 & - & - \\
\hline Acute infection (pneumonia) & 11 & - & - \\
\hline latrogenic (AVP analogs) & 4 & - & - \\
\hline Idiopathic & 10 & - & - \\
\hline Extracellular volume depletion & 0 & 13 & 4 \\
\hline Euvolemia & 42 & 7 & 17 \\
\hline Extracellular volume expansion & 0 & 5 & 2 \\
\hline$\%$ of current diuretic therapy (mdd, days) & $12(28.6 \%)$ & $25(100 \%)$ & $22(100 \%)$ \\
\hline Furosemide (20 mg, $3.2 \mathrm{~d}$ ) & 8 & 19 & 0 \\
\hline Furosemide + canrenoate $(45 \mathrm{mg}+90 \mathrm{mg}, 3.0 \mathrm{~d})$ & 4 & 6 & 0 \\
\hline Hydrochlorothiazide (17.1 mg, 4.3 d) & 0 & 0 & 17 \\
\hline Indapamide (3.8 mg, $5.1 \mathrm{~d})$ & 0 & 0 & 3 \\
\hline Metolazone (4.1 mg, $3.8 \mathrm{~d}$ ) & 0 & 0 & 2 \\
\hline
\end{tabular}

\section{TABLE 1: Clinical characteristics of the investigated groups.}

SIAD: Syndrome of inappropriate antidiuresis; DIH: Diuretic-induced hyponatremia; TIH: Thiazide-induced hyponatremia; AVP: Vasopressin analogs. (mdd, days) indicate the mean daily dose of diuretics and mean time of withdrawal of the diuretic therapy to obtain normonatraemia. Data are mean \pm standard deviation or numbers.

DIH and TIH patients were older compared with SIAD patients. Diuretic therapy was present in $28.6 \%$ of the SIAD patients where the dominant cause was neoplasia (17/42 patients) and infections, usually pneumonia (11/42 patients). In DIH patients vs. TIH the extracellular volume depletion was a dominant finding (13/25 vs. $4 / 22$ patients). The fluid challenge test was performed in 29 patients (12 patients identified as SIAD, 13 patients as DIH and four patients as TIH). A significant difference was found among SIAD and diuretictreated patients in BUN, serum UA and urine Na value while FEUA was significantly different only in SIAD vs. DIH patients (Table 2). 


\section{Cureus}

\begin{tabular}{|c|c|c|c|c|}
\hline & SIAD & DIH & TIH & P-value \\
\hline \multicolumn{5}{|l|}{ Serum } \\
\hline $\mathrm{Na}(135-145$ mEq/L) & $126.6(6.9)$ & $128.4(2.8)$ & $127.4(5.8)$ & NS \\
\hline $\mathrm{K}(3.5-4.5 \mathrm{mEq} / \mathrm{L})$ & $4.1(0.5)$ & $3.8(0.8)$ & $4.2(1.1)$ & NS \\
\hline Creatinine (0.5-1.2 mg/dl) & $0.5(0.2)$ & $1.0(0.4)$ & $0.9(0.3)$ & NS \\
\hline BUN (20-50 mg/dl) & $24.2(10.4)$ & $55.8(21.8)^{\star}$ & $53.1(32.3)^{\star}$ & 0.01 \\
\hline UA (3.4-7.0 mg/dl) & $2.5(1.1)$ & $8.0(3.6)^{\star}$ & $4.4(2.2)^{*}$ & 0.001 \\
\hline Osmolality (275-285 mEq/kg) & $254.3(12)$ & $263.8(15)$ & $261.4(13)$ & NS \\
\hline \multicolumn{5}{|l|}{ Urine } \\
\hline $\mathrm{Na}(100-200$ mEq/L) & $105.9(78)$ & $37.3(29.9)^{\star}$ & $51.7(43.2)^{\star}$ & 0.001 \\
\hline K (50-100 mEq/L) & $28.0(22.7)$ & $33.0(4.1)$ & $27.8(14.9)$ & NS \\
\hline Osmolality (50-1200 mEq/kg) & $487.3(167)$ & $497.6(151.7)$ & 476.6 (194) & NS \\
\hline \multicolumn{5}{|l|}{ FE } \\
\hline $\mathrm{Na}(\%)$ & $1.6(0.4)$ & $1.4(0.6)$ & $1.2 / 0.6)$ & NS \\
\hline UA (5-9\%) & $16.1(4.4)$ & $5.9(3.8)^{\star}$ & $14.6(6.6)$ & 0.001 \\
\hline
\end{tabular}

TABLE 2: Laboratory parameters in the investigated study groups.

Data are mean (standard deviation) or numbers.

* Indicates a P-value $<0.05$ vs. SIAD.

SIAD: Syndrome of inappropriate antidiuresis; DIH: Diuretic-induced hyponatremia; TIH: Thiazide-induced hyponatremia; FE: Fractional excretion.

The ROC analysis confirmed that FEUA discriminated better than sUA between SIAD and DIH patients (AUC $0.96,<0.001$ vs. $0.88,<0.001)$ while it was a poor marker to discriminate between SIAD and TIH $(0.65$, NS vs. 0.67 , NS, Figure 1).

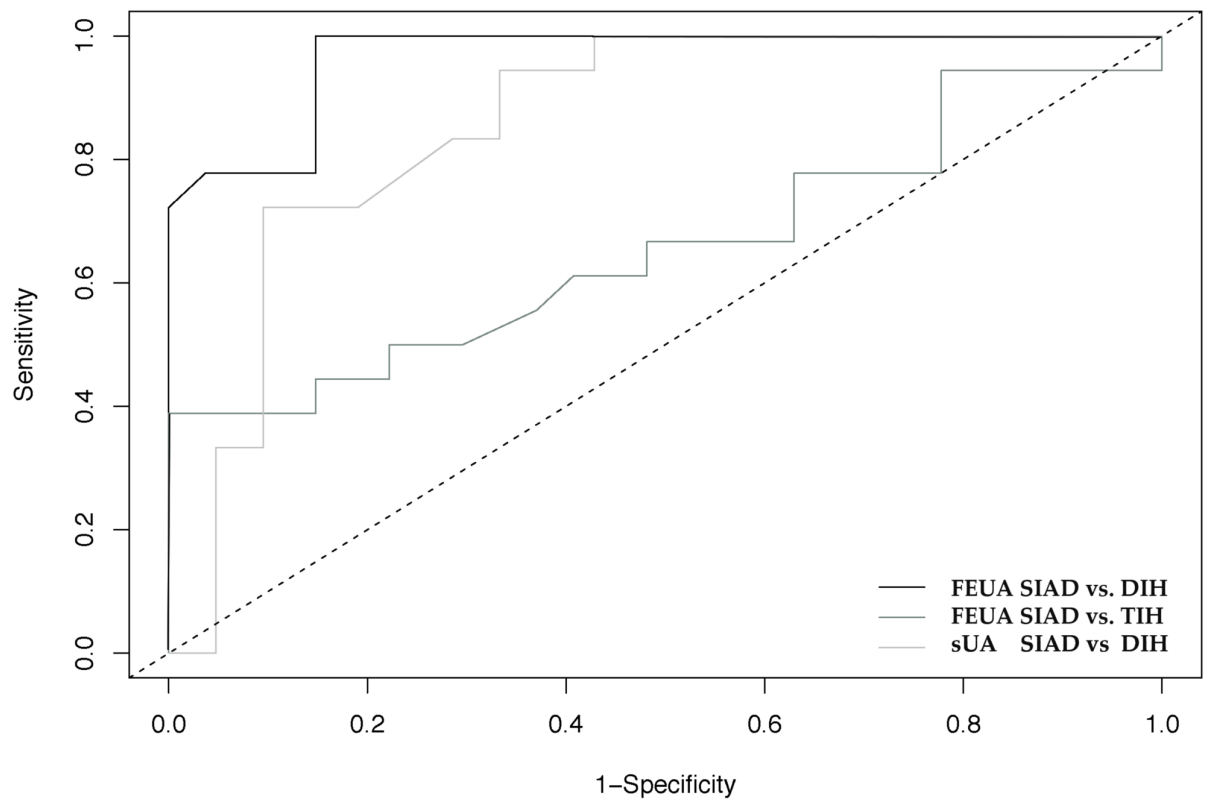

FIGURE 1: ROC analysis of the three investigated groups.

Area under the curve $(95 \% \mathrm{Cl})$ 
Comparison of the diagnostic utility (ROC analysis, area under curve: AUC) of fractional excretion of uric acid (FEUA) to differentiate among syndrome of inappropriate antidiuresis (SIAD) and diuretic-induced

hyponatremia (DIH) or thiazide-induced hyponatremia (TIH) patients and serum uric acid (sUA) between SIAD and DIH. The diagonal line indicates the area of 0.5 , corresponding to no informative discrimination.

The sensitivity analysis showed that FEUA cut-off point higher than $10 \%$ resulted in a $100 \%$ specificity and $85 \%$ sensitivity while a value less than $8 \%$ showed a $100 \%$ sensitivity and $72 \%$ specificity to identify SIAD patients (Table 3).

\begin{tabular}{|c|c|c|c|}
\hline FEUA & $8 \%$ & $9 \%$ & $10 \%$ \\
\hline Sensitivity & $100 \%$ & $85 \%$ & $85 \%$ \\
\hline Specificity & $72 \%$ & $77 \%$ & $100 \%$ \\
\hline
\end{tabular}

\section{TABLE 3: FEUA cut off points.}

Different FEUA cut off points with relative sensitivity and specificity. Normal euvolemic population shows a $5-9 \%$ range.

FEUA: Fractional excretion of uric acid.

\section{Discussion}

Hyponatraemia is by far the most common electrolyte imbalance found in hospital population and a severe grade $(<125 \mathrm{mEq} / \mathrm{L})$ is detected in $2 \%$ of inpatients [2]. The natriuretic effect of diuretic therapy has a confounding role in the evaluation of EABV to obtain a differential diagnosis of hyponatraemia investigated with different algorithms. Usually, thiazide drugs increase six-fold the risk of hyponatraemia vs. nonexposed patients with an estimated incidence of $11 \%$ in the geriatric population [14]. The causes of TIH, beyond the tubular effects of thiazides on specific $\mathrm{Na} / \mathrm{Cl}$ cotransporters, are water retention, both with an increase of arginine vasopressin activity and/or upregulation of aquaporin-2 expression, resembling a laboratory pattern of SIAD $[15,16]$. Furthermore, hypokalaemia, a frequent hallmark in thiazide-treated patients, inducing potassium depletion can increase the volume receptor release of vasopressin [17]. However, most patients in our study did not show associated hypokalaemia, probably in consideration of the low daily dose of thiazide therapy. Another possible key to explain TIH could be the genetic expression of specific prostaglandin transporters in a subset of patients prone to develop hyponatraemia [18, 19].

According to these data, the majority of patients affected by TIH are clinically euvolemic showing characteristics of extracellular volume expansion [20]. In the real world, the withdrawal of diuretics is generally considered mandatory for a correct diagnosis resulting in a longer hospitalization period and increased hospital admission costs.

Serum UA and its fraction excretion are considered useful markers of the volemic status in acute patients being on UA renal handling in the proximal tubule preserved by interferences induced by the most used diuretics [21]. Moreover, FEUA value on a urine spot sample has the advantage to avoid a 24-h urine collection resulting in a more accurate parameter [22]. Beck reported that hyponatraemia secondary to SIADH is generally associated with a serum uric acid level $<4 \mathrm{mg} / \mathrm{dl}$ with an increase of FEUA [23]. These findings are dependent on a decrease in tubular reabsorption of urate whereas secretion seems to be appropriate for the low level of uricemia [24, 25].

Fenske et al. have demonstrated that FEUA cut-off value >12\% (86\% sensitivity and $100 \%$ specificity) is an optimal marker to discriminate between SIAD and diuretic-induced hyponatraemia. The limit of his study was the reduced number of patients treated with thiazide (only seven patients corresponding to $13 \%$ of the overall group) [5].

Our results confirm that FEUA value is a reliable marker to discriminate SIAD vs. sodium depleted DIH patients excluding the need for diuretic withdrawal and/or a fluid challenge test with a risk of water overload. The sensitivity analysis shows that FEUA cutoff point higher than $10 \%$ confirms SIAD diagnosis (100\% specificity) whereas a value of less than $8 \%$ excludes it (100\% sensitivity).

But instead, FEUA value is not able to identify correctly TIH vs. SIAD patients in consideration that the majority of our investigated TIH patients show effectively a euvolemic phenotype resembling SIAD pattern (17/22 patients, $77 \%)$. 
Limitation of our study includes the small numbers of studied patients and we need further investigation to confirm the real utility in the diagnostic approach of hyponatremia.

\section{Conclusions}

In conclusion, our data confirm that a calculated parameter such as FEUA is an excellent marker to discriminate at admission SIAD vs. sodium depleted patients treated with furosemide and/or potassium canrenoate by avoiding the need for diuretic withdrawal or fluid challenge test. So otherwise, thiazide withdrawal, beyond obtaining a serum Na normalization, is still mandatory for differential diagnosis of the hyponatraemia in TIH vs. SIAD patients.

\section{Additional Information \\ Disclosures}

Human subjects: Consent was obtained by all participants in this study. Animal subjects: All authors have confirmed that this study did not involve animal subjects or tissue. Conflicts of interest: In compliance with the ICMJE uniform disclosure form, all authors declare the following: Payment/services info: All authors have declared that no financial support was received from any organization for the submitted work. Financial relationships: All authors have declared that they have no financial relationships at present or within the previous three years with any organizations that might have an interest in the submitted work. Other relationships: All authors have declared that there are no other relationships or activities that could appear to have influenced the submitted work.

\section{References}

1. Upadhyay A, Jaber BL, Madias NE: Incidence and prevalence of hyponatremia. Am J Med. 2006, 119:30-35. 10.1016/j.amjmed.2006.05.005

2. Verbalis JG: The syndrome of inappropriate antidiuretic hormone secretion and other hyposmolar disorders . In: Diseases of the Kidney and Urinary Tract. Schrier RW (ed): Lippincott Williams \& Wilkins, Philadelphia; 2006. 2511-2548

3. Verbalis JG, Goldsmith SR, Greenberg A, Schrier RW, Sterns RH: Hyponatremia treatment guidelines 2007: expert panel recommendations. Am J Med. 2007, 120:1-21. 10.1016/j.amjmed.2007.09.001

4. Lien YH, Shapiro JI: Hyponatremia: clinical diagnosis and management. Am J Med. 2007, 120:653-658. 10.1016/i.amimed.2006.09.031

5. Fenske W, Störk S, Koschker AC, Blechschmidt A, Lorenz D, Wortmann S, Allolio B: Value of fractional uric acid excretion in differential diagnosis of hyponatremic patients on diuretics. J Clin Endocrinol Metab. 2008, 93:2991-2997. 10.1210/jc.2008-0330

6. Chung HM, Kluge R, Schrier RW, Anderson RJ: Clinical assessment of extracellular fluid volume in hyponatremia. Am J Med. 1987, 83:905-908. 10.1016/0002-9343(87)90649-8

7. Decaux G, Prospert F, Cauchie P, Soupart A: Dissociation between uric acid and urea clearances in the syndrome of inappropriate secretion of antidiuretic hormone related to salt excretion. Clin Sci (Lond). 1990, 78:451-455. 10.1042/cs0780451

8. Musch W, Hedeshi A, Decaux G: Low sodium excretion in SIADH patients with low diuresis . Nephron Physiol. 2004, 96:11-18. 10.1159/000075575

9. Fichman MP, Vorherr H, Kleeman CR, Telfer N: Diuretic-induced hyponatremia. Ann Intern Med. 1971, 75:853-863. 10.7326/0003-4819-75-6-853

10. Decaux G, Dumont I, Naeije N, Mols P, Melot C, Mockel J: High uric acid and urea clearance in cirrhosis secondary to increased "effective vascular volume". Am J Med. 1982, 73:328-334.

11. Maesaka JK, Gupta S, Fishbane S: Cerebral salt-wasting syndrome: does it exist?. Nephron. 1999, 82:100109. $10.1159 / 000045384$

12. McGee S, Abernethy WB 3rd, Simel DL: Is this patient hypovolemic? . JAMA. 1999, 281:1022-1029. 10.1001/jama.281.11.1022

13. Schwartz WB, Bennett W, Curelop S, Bartter FC: A syndrome of renal sodium loss and hyponatremia probably resulting from inappropriate secretion of antidiuretic hormone. Am J Med. 1957, 23:529-542. 10.1016/0002-9343(57)90224-3

14. Byatt CM, Millard PH, Levin GE: Diuretics and electrolyte disturbances in 1000 consecutive geriatric admissions. J R Soc Med. 1990, 83:704-708.

15. Reilly RF, Ellison DH: Mammalian distal tubule: physiology, pathophysiology, and molecular anatomy . Physiol Rev. 2000, 80:277-313. 10.1152/physrev.2000.80.1.277

16. Kim GH, Lee JW, Oh YK, et al.: Antidiuretic effect of hydrochlorothiazide in lithium-induced nephrogenic diabetes insipidus is associated with upregulation of aquaporin-2, $\mathrm{Na}-\mathrm{Cl}$ co-transporter, and epithelial sodium channel. J Am Soc Nephrol. 2004, 15:2836-2843. 10.1097/01.ASN.0000143476.93376.04

17. Fuisz RE, Lauler DP, Cohen P: Diuretic-induced hyponatremia and sustained antidiuresis . Am J Med. 1962, 33:783-791. 10.1016/0002-9343(62)90255-3

18. Channavajihala SK, Bramley R, Peltz T, et al.: Urinary extracellular vesicle protein profiling and endogenous lithium clearance support excessive renal sodium wasting and water reabsorption in thiazide-induced hyponatremia. Kidney Int Rep. 2019, 4:139-147. 10.1016/j.ekir.2018.09.011

19. Ware JS, Wain LV, Channavajihala SK, et al.: Phenotypic and pharmacogenetic evaluation of patients with thiazide-induced hyponatremia. J Clin Invest. 2017, 127:3367-3374. 10.1172/JCI89812

20. Sonnenblick M, Rosin AJ: Significance of the measurement of uric acid fractional clearance in diureticinduced hyponatraemia. Postgrad Med J. 1986, 62:449-452. 10.1136/pgmj.62.728.449

21. Edwards A, Auberson M, Ramakrishnan SK, Bonny O: A model of uric acid transport in the rat proximal 


\section{Cureus}

tubule. Am J Physiol Renal Physiol. 2019, 316:934-947. 10.1152/ajprenal.00603.2018

22. Elisaf M, Siamopoulos KC: Fractional excretion of potassium in normal subjects and in patients with hypokalaemia. Postgrad Med J. 1995, 71:211-212. 10.1136/pgmj.71.834.211

23. Beck LH: Hypouricemia in the syndrome of inappropriate secretion of antidiuretic hormone . N Engl J Med. 1979, 301:528-530. 10.1056/NEJM197909063011005

24. Decaux G, Dumont I, Waterlot Y, Hanson B: Mechanisms of hypouricemia in the syndrome of inappropriate secretion of antidiuretic hormone. Nephron. 1985, 39:164-168. 10.1159/000183365

25. Prospert F, Soupart A, Brimioulle S, Decaux G: Evidence of defective tubular reabsorption and normal secretion of uric acid in the syndrome of inappropriate secretion of antidiuretic hormone. Nephron. 1993, 64:189-192. 10.1159/000187312 